\title{
Pediatric-Onset Refractory Lupus Erythematosus Panniculitis Treated With Rituximab
}

\author{
Divya Angra, MD; Philip A. Roehrs, MD; Paul B. Googe, MD; Adewole S. Adamson, MD, MPP
}

\section{PRACTICE POINTS}

- Lupus erythematosus panniculitis (LEP) is rare in the pediatric population and often is difficult to treat.

- Rituximab can be an effective treatment option for refractory LEP.

- Before the initiation of rituximab, a biopsy is warranted to rule out subcutaneous T-cell lymphoma, which can mimic LEP and hemophagocytic lymphohistiocytosisassociated panniculitis.

To the Editor:

Lupus erythematosus panniculitis (LEP) is rare in the pediatric population. It can be difficult to manage, as patients may not respond to conventional treatments including hydroxychloroquine and prednisone. We report the use of rituximab in the treatment of a 20-year-old woman with LEP of the face, legs, and arms that was refractory to standard treatments. She also had a history of hemophagocytic lymphohistiocytosis (HLH). Further studies are warranted to determine the role of rituximab in the treatment of pediatric patients with LEP.

A 20-year-old woman with history of LEP and HLH initially presented with migratory violaceous nodules on the face 16 years prior to the current presentation. A skin biopsy 3 years after that initial presentation suggested a diagnosis of cutaneous lupus erythematosus. Six years later, numerous asymptomatic lesions appeared on the legs, predominantly on the calves; she was successfully treated with hydroxychloroquine and high-dose prednisone. Four years prior to the current presentation, a febrile illness prompted discontinuation of hydroxychloroquine and hospitalization, where she was first was diagnosed with HLH; she achieved remission with cyclosporine. At the current presentation, she continued to have persistent violaceous lesions on the face, lower arms, and legs with underlying nodularity (Figure 1). Skin biopsies revealed LEP and were less suggestive of HLH. She was restarted on hydroxychloroquine, which did not adequately control

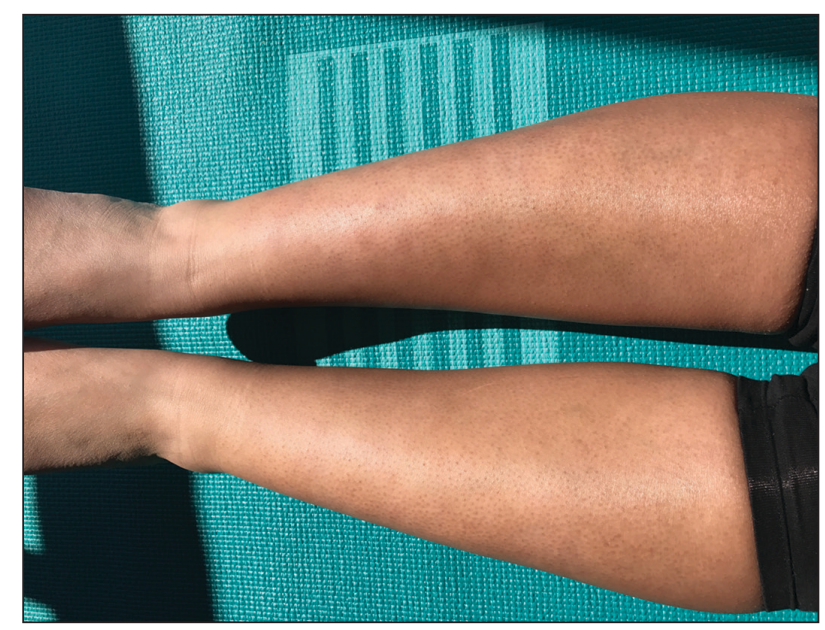

FIGURE 1. Violaceous patches of lupus erythematosus panniculitis on the lower legs prior to rituximab treatment.

Dr. Angra is in private practice, Alexandria, Virginia. Dr. Roehrs is from the Department of Pediatrics, Division of Hematology and Oncology, University of Virginia Health System, Charlottesville. Dr. Googe is from the Department of Dermatology, University of North Carolina Health Care System, Chapel Hill. Dr. Adamson is from Dell Medical School, University of Texas, Austin. 
the disease. Rheumatologic workup was only notable for an antinuclear antibody titer of 1:80 (reference range, $<1: 80)$ in a speckled pattern.

Due to the refractory nature of her condition, continued lesion development despite standard treatment, and concerns of possible scarring, we considered a trial of rituximab. Because HLH and LEP can mimic subcutaneous T-cell lymphoma, another skin biopsy was performed, which revealed a deep dermal and subcutaneous lymphohistiocytic infiltrate composed of predominantly $\mathrm{CD}^{+} \mathrm{T}$ cells with a mixed population of $\mathrm{CD}^{+}$and $\mathrm{CD} 8^{+}$cells (Figure 2). There was no evidence of transformation into lymphoma. Pathologic findings were most compatible with LEP rather than an HLH-associated panniculitis due to the lack of definitive phagocytosis. She received rituximab using body surface areabased dosing at $375 \mathrm{mg} / \mathrm{m}^{2}$. CD19 levels decreased to
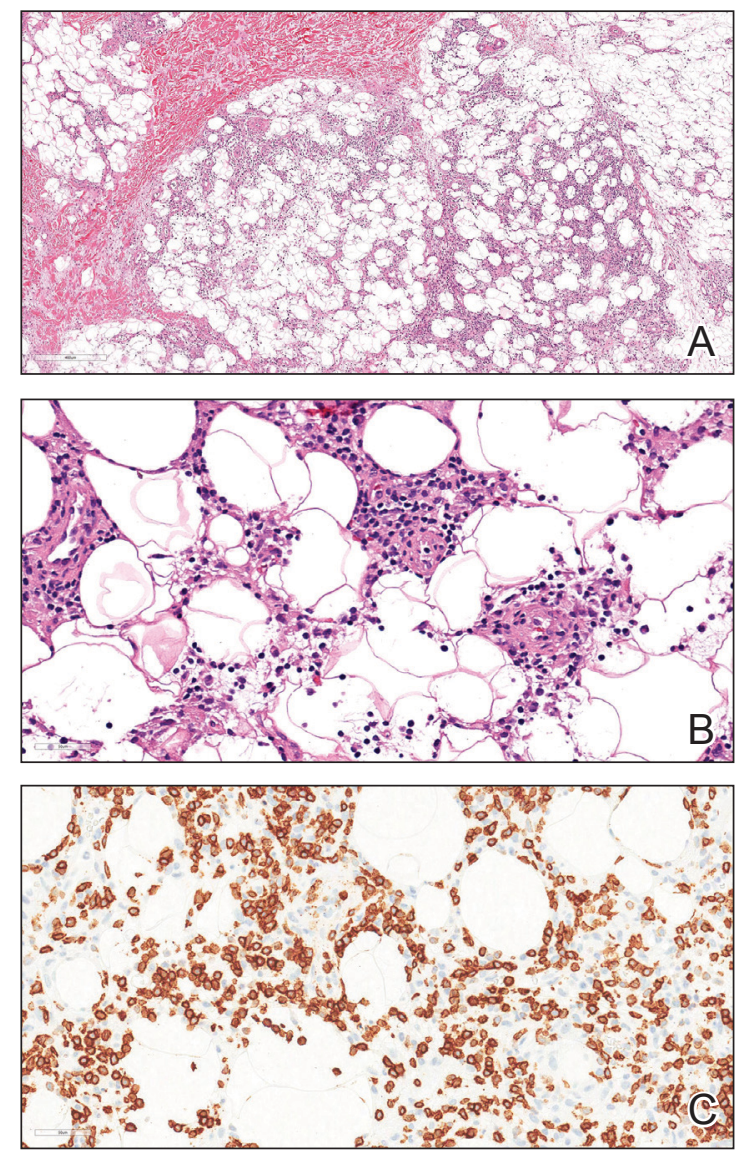

FIGURE 2. A, An excisional biopsy of the skin inclusive of subcutaneous fat showed a lobular infiltrate of mononuclear leukocytes with no vasculitis or granulomas (H\&E, original magnification $\times 40$ ). B, Lymphocytes, occasional plasma cells, and few histiocytes filled the interstitial spaces in the lobule of subcutaneous fat. There was no lymphocyte atypia, necrosis, or ringing of adipocytes by lymphocytes (H\&E, original magnification $\times 400)$. C, The lymphocytes were $T$ cells that stained positively with CD3 with diaminobenzidine immunoperoxidase. There was a mixture of $\mathrm{CD}^{+}{ }^{+}$and $\mathrm{CD} 8^{+}$cells with a predominance of CD4 cells (original magnification $\times 400$ ). undetectable levels after the first dose. Rituximab was dosed based on clinical response; she tolerated treatment well and experienced considerable improvement in the number of lesions following completion of 4 doses at weeks $0,1,5$, and 7 (Figure 3). She developed a flare at 7 months and improved again after another dose of rituximab.

Lupus erythematosus panniculitis is a rare variant of lupus erythematosus with an average age of presentation between 30 and 60 years. ${ }^{1}$ In children, LEP presents as recurrent subcutaneous nodules and plaques, commonly involving the face and upper arms. ${ }^{1,2}$ Long-term sequelae include local swelling and skin atrophy. ${ }^{3}$ Conventional treatment options for pediatric patients include hydroxychloroquine and corticosteroids. Management can be challenging due to the lack of response to conventional treatments as well as the chronic progressive nature of LEP. ${ }^{2}$ In refractory cases, cyclosporine, azathioprine, sulfones, thalidomide, mycophenolate mofetil, and cyclophosphamide are alternative treatment options..$^{1-4}$

Rituximab, a chimeric monoclonal antibody targeting B-cell surface marker CD20, results in depletion of mature B cells. Use of rituximab for LEP has been described in multiple case reports involving an 8-yearold boy, 22-year-old girl, and 2 middle-aged women. ${ }^{2-4}$ In addition, a recently published case series of 4 patients with childhood-onset refractory LEP described improvement of disease activity with rituximab. ${ }^{5}$ It is important to rule out subcutaneous T-cell lymphoma before treatment with rituximab, as its histopathology can closely resemble that seen in LEP and HLH-associated cytophagic histiocytic panniculitis. ${ }^{1,6}$

Rituximab may be an effective treatment option in pediatric patients with refractory LEP. Larger studies on the use of rituximab in the pediatric population are necessary.

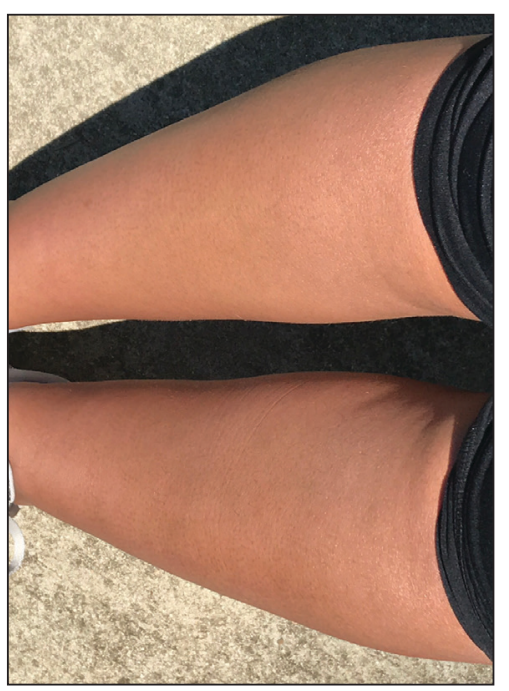

FIGURE 3. Improvement in violaceous patches of lupus erythematosus panniculitis following rituximab treatment. 


\section{REFERENCES}

1. Weingartner JS, Zedek DC, Burkhart CN, et al. Lupus erythematosus panniculitis in children: report of three cases and review of previously reported cases. Pediatr Dermatol. 2011;29:169-176.

2. Moreno-Suárez F, Pulpillo-Ruiz Á. Rituximab for the treatment of lupus erythematosus panniculitis. Dermatol Ther. 2013;26:415-418.

3. Guissa VR, Trudes G, Jesus AA, et al. Lupus erythematosus panniculitis in children and adolescents. Acta Reumatol Port. 2012;37:82-85.
4. Mcardle A, Baker JF. A case of "refractory"lupus erythematosus profundus responsive to rituximab. Clin Rheumatol. 2009;28:745-746.

5. Correll CK, Miller DD, Maguiness SM. Treatment of childhoodonset lupus erythematosus panniculitis with rituximab. JAMA Dermatol. 2020;156:566-569.

6. Aronson IK, Worobec SM. Cytophagic histiocytic panniculitis and hemophagocytic lymphohistiocytosis: an overview. Dermatol Ther. 2010;23:389-402. 